\title{
Margin Call: an Accessible Web-based Text Viewer with Generated Paragraph Summaries in the Margin
}

\author{
Naba Rizvi ${ }^{1,2}$, Sebastian Gehrmann ${ }^{2,3}$, Lidan Wang ${ }^{2}$, Franck Dernoncourt ${ }^{2}$ \\ ${ }^{1}$ University of Toledo, ${ }^{2}$ Adobe Research, ${ }^{3}$ Harvard SEAS \\ nabah.rizvi@rockets.utoledo.edu, gehrmann@seas.harvard.edu, \\ lidwang@adobe.com, franck. dernoncourteadobe.com
}

\begin{abstract}
We present Margin Call, an accessible webbased text viewer that automatically generates short summaries for each paragraph of the text and displays the summaries in the margin of the text next to the corresponding paragraph. On the back-end, the summarizer first identifies the most important sentence for each paragraph in the text file uploaded by the user. The selected sentence is then automatically compressed to produce the short summary. The resulting summary is a few words long. The displayed summaries can help the user understand and retrieve information faster from the text, while increasing the retention of information.
\end{abstract}

\section{Introduction}

Long documents can be difficult and timeconsuming to read and comprehend, especially for people with reading or visual impairments. Before deciding whether to read a document or to find some information faster, it may be helpful to have an overview first. However, this is not possible with screen readers or without advanced skimming/reading skills. Moreover, it is easy to lose track of the overall story and message. Previous work showed that presenting a brief summary for each section of a text can help readers understand a text better (Kintsch and Van Dijk, 1978; Wiley and Rayner, 2000; Gehrmann et al., 2019), increase the reading speed (Bransford and Johnson, 1972) and improve the recall of content (Dooling and Lachman, 1971; Smith and Swinney, 1992).

To that end, we introduce in this work a web-based assistive text viewer that presents a document in an accessible way. The tool is accessible online at https://github.com/ Franck-Dernoncourt/margincall.

\section{Margin Call}

Margin Call is an open-source web-based text viewer that provides the user with automatically generated summaries obtained from individual paragraphs in a text. It uses an extractive summarizer to obtain the best sentence for each paragraph, which is then compressed using a deletionbased sentence compressor.

\subsection{Model}

A common approach for text summarization uses information obtained from the content of a sentence to classify them as a whole (Cheng and Lapata, 2016; Dlikman and Last, 2016). This approach has limitations when using traditional hand-crafted feature functions and requires powerful sentence representations when applying a datadriven approach. Margin Call addresses this issue by scoring sentences on a word-level while still extracting full sentences. Margin Call uses the sentence extracted from the extractive summarizer and passes it to a deletion-based compressor. The compressor is based on a neural SemiMarkov Conditional Random Field, which decides whether a word should be included in the compressed output.

\subsection{User Interface}

Margin Call displays the generated summary next to each corresponding paragraph and highlights the sentence in the paragraph the summary was extracted from, as shown in Figure 1. This lets the user know which sentence in the paragraph was used to produce the summary.

\subsection{Accessibility}

Margin Call was developed to be accessible for users with various visual impairments. Research has shown color-blindness has an impact on the 


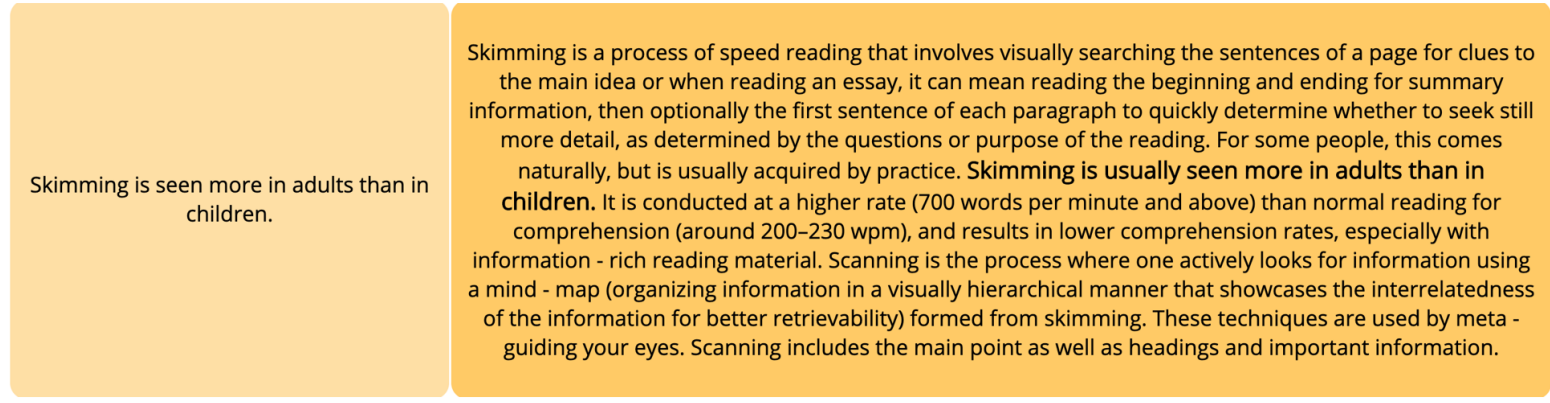

\section{Summary Only}

Speed claims have been controversial.

Figure 1: The interface of Margin Call displays summaries generated for each paragraph, allowing the user to decide to view the summary with the text it is generated from or by itself.

readability of web applications (Sparks, 2019). When developing the program, we decided to display the values in an HTML table to make the content easy for screen readers to interpret. The extension ChromeVox was used to test how a blind user would experience the website with the aid of a screen reader.

\subsection{Implementation}

The summarization models are implemented in PyTorch, the web interface relies on Flask, and the application is packaged as a Docker image.

\section{Conclusion and Future Work}

Margin Call is an easy-to-use and accessible web application that summarizes each paragraph of a text, thereby making state-of-the-art summarization techniques available to anyone. The summaries are displayed next to each paragraph, which help the reader understand, retrieve and memorize the information faster.

As extension of Margin Call, one could explore automatically segmenting text into paragraphs in case the text does not contain any paragraph, or if a paragraph is too long. Another improvement could be to allow the user to add some bias reflecting which aspect(s) of the text (e.g., "science" or "financial markets") they care the most about, so that the generated summaries reflect the user's interests.

\section{References}

John D Bransford and Marcia K Johnson. 1972. Contextual prerequisites for understanding: Some investigations of comprehension and recall. Journal of verbal learning and verbal behavior, 11(6):717726.

Jianpeng Cheng and Mirella Lapata. 2016. Neural summarization by extracting sentences and words. Proceedings of the 54th Annual Meeting of the Association for Computational Linguistics (Volume 1: Long Papers).

Alexander Dlikman and Mark Last. 2016. Using machine learning methods and linguistic features in single-document extractive summarization. DMNLP@PKDD/ECML, page 18.

D James Dooling and Roy Lachman. 1971. Effects of comprehension on retention of prose. Journal of experimental psychology, 88(2):216.

Sebastian Gehrmann, Steven Layne, and Franck Dernoncourt. 2019. Improving human text comprehension through semi-Markov CRF-based neural section title generation. In Proceedings of the 2019 Conference of the North American Chapter of the Association for Computational Linguistics: $\mathrm{Hu}$ man Language Technologies, Volume 1 (Long and Short Papers), pages 1677-1688, Minneapolis, Minnesota. Association for Computational Linguistics.

Teun A van DijkWalter Kintsch and TA Van Dijk. 1978. Cognitive psychology and discourse: Recalling and summarizing stories. Current trends in textlinguistics, page 61 .

Edward E Smith and David A Swinney. 1992. The role of schemas in reading text: A real-time examination. Discourse Processes, 15(3):303-316. 
Theresa Marie Sparks. 2019. The effects of color choice in web design on the usability for individuals with color-blindness.

Jennifer Wiley and Keith Rayner. 2000. Effects of titles on the processing of text and lexically ambiguous words: Evidence from eye movements. Memory \& Cognition, 28(6):1011-1021. 